\title{
Risk Factors and Options of Management for an Incidental Dural Tear in Biportal Endoscopic Spine Surgery
}

\author{
Ju-Eun Kim ${ }^{1}$, Dae-Jung Choi ${ }^{1}$, Eugene J. Park ${ }^{2}$ \\ ${ }^{1}$ Department of Orthopedic Surgery, Himnaera Hospital, Busan, Korea \\ ${ }^{2}$ Department of Orthopedic Surgery, Chungnam National University College of Medicine, Daejeon, Korea
}

\begin{abstract}
Study Design: Here we perform a retrospective analysis regarding an incidental dural tear (IDT) during biportal endoscopic spinal surgery (BESS).

Purpose: This study investigates the causes of IDT specifically related to technical procedures of BESS with the aim of lowering its risk during training.

Overview of Literature: The incidence of dural tear is reported $0.5 \%-18 \%$ in open spinal surgery and $1.7 \%-4.3 \%$ during endoscopic spinal surgery. Because conversion to open surgery for direct repair could become necessary during endoscopic spinal surgery, prevention of this complication is essential.

Methods: We have retrospectively studied IDTs by four surgeons during 1 or 2 years after starting BESS for lumbar degenerative diseases and analyzed the locations, sizes, and specific endoscopic conditions specific to each.

Results: Twenty-five cases (1.6\%) of IDTs among 1,551 cases of BESS occurred; 13 cases (52\%) of these were within the first 6 months. The locations were dorsal midline in 12 cases, ipsilateral side in 11 cases, and contralateral side in two cases. The tear sizes were $<10 \mathrm{~mm}$ in 20 cases and $\geq 10 \mathrm{~mm}$ in five cases. IDT commonly occurred due to injury of central dural folding during flavectomy under turbid surgical fields due to small bleeds under water. Twenty cases with IDTs of $<10 \mathrm{~mm}$ were treated well with the patch technique. Among five cases of $\geq 10 \mathrm{~mm}$, three underwent open repair within a few days, and two of these which failed to conservative management required a delayed revision operation due to pseudomeningocele. No cases progressed to surgical site infection or infectious spondylitis.

Conclusions: IDTs of $<10 \mathrm{~mm}$ can be successfully treated with the patch technique. To prevent IDT during the early learning period, maintaining clear visibility by securing fluent saline outflow and meticulous hemostasis of small bleeding from exposed cancellous bone and epidural vessels is essential with caution not to injure the central dural folding during midline flavectomy.
\end{abstract}

Keywords: Biportal endoscopic spine surgery; Incidental dural tear; Risk factors; Lumbosacral lesion

\section{Introduction}

Biportal endoscopic spinal surgery (BESS) is widely per- formed as a minimally invasive surgery (MIS) technique for lumbar spinal disease [1-5]. Although the followup period for this is short, BESS can be applied not only

Received Sep 8, 2019; Revised Nov 24, 2019; Accepted Dec 8, 2019

Corresponding author: Dae-Jung Choi

Department of Orthopedic Surgery, Himnaera Hospital, 85 Beomil-ro, Dong-gu, Busan 48735, Korea

Tel: +82-55-711-9500, Fax: +82-55-711-9505, E-mail: djchoi9@hanmail.net 
to repair lumbar disc herniation [6] but also for lumbar spinal stenosis with improvement of clinical and radiological outcomes $[1,3]$. BESS had widened indications for patients with segmental instability or degenerated lumbar disease with foraminal stenosis in hopes of preserving back muscle and facet integrity [5]. Despite its excellent short-term clinical outcomes, however, BESS has shown learning-curve-related complications such as incidental dural tear (IDT), incomplete decompression, and postoperative hematoma [7].

IDT is one of the most common complications in microscopic spine surgery, and the reported incidence of IDT is $0.5 \%-18 \%$ in lumbar spinal surgery and $2.9 \%$ in BESS [7-12]. IDT is considered a minor complication due to small risk of neurological sequelae when it is well treated. Nevertheless, it may cause chronic lower back pain because proper IDT repair may require greater muscle dissection or wider laminectomy. Sometimes a surgeon opts to reduce surgery time for an IDT, risking incomplete decompression of the spine. Furthermore, if an IDT is not managed appropriately, further complications such as pseudomeningocele due to cerebrospinal fluid (CSF) leakage, surgical site infection, or meningitis could occur. These complications lead to increased physical and financial burdens to the patient [13].

Primary repair of a dural tear is the currently the gold standard for treating IDT during conventional spinal surgery $[14,15]$. Endoscopic spine surgery, however, has no standard treatment protocol to cope with IDT. Common strategies include conversion to open repair with discontinuation of minimally invasive spine surgery or conservative management with a delayed decision depending on the state of sequelae. Because BESS is a new technique, recruited surgeons must struggle to overcome the learningcurve period of an unfamiliar endoscopic view that is under water. This report is focused on the common causes of IDT during BESS and reviews the previous literature regarding IDT in lumbar spine surgery to suggest a standardized treatment protocol to repair IDT during BESS.

\section{Materials and Methods}

The purpose of this study is for retrospectively reviewing the technical procedures and management of IDT during new endoscopic spine surgery, not for case controlled study. So, this study has proceeded without the need of permitting the Institutional Review Board. We reviewed the cases in which IDT occurred during BESS for degenerative lumbar diseases including spinal stenosis, degenerative spondylolisthesis, adult isthmic spondylolisthesis, facet cyst, disc herniation, and revision surgery at the same level by four surgeons. A total of 1,551 cases were included from the beginning of BESS training by each surgeon for 1 or 2 years, and the early learningcurve period was defined as the first 6 months. Surgeon 1 performed 768 cases of BESS during the study period, surgeon 2 performed 253 cases, surgeon 3 performed 130 cases, and surgeon 4 performed 400 cases. The statistical differences of the incidence of IDT before or after the 6-month learning-curve period among all four surgeons was analyzed by chi-square test using IBM SPSS Software ver. 24.0 (IBM Corp., Armonk, NY, USA).

Among the 1,511 cases that underwent either decompression or discectomy by BESS, 25 cases (1.6\%) of IDT were retrospectively reviewed by observing recorded operating video clips and medical records to analyze the parameters of each IDT. These were classified by size (small-sized tear of $<1 \mathrm{~cm}$ or large-sized tear of $\geq 1 \mathrm{~cm}$ ), location (center midline or lateral side), morphology (slit tear or flap tear), treatment methods (patch compression method, immediate open repair, or delayed conversion to open repair), and final clinical results.

The patch compression method was applied for small IDTs of $<1 \mathrm{~cm}$. Takeda (Linz, Austria; a fibrin-based hemostat) and Gelfoam (Ethicon, NJ, USA) were cut into 1 $\mathrm{cm}^{2}$ pieces. A piece of TachoSil was patched on the tear site, and Gelfoam was placed over it. Meticulous control for small bleeding from peripheral muscle ends was performed using small-headed radiofrequency. Leaving only a small amount of hematoma was enough to decrease dural pulsation by compressing the patched site (Fig. 1). The surgical wounds of the cases being treated with patch compression could not be sutured tightly at the muscle layer through small endoscopic wounds, so the subcutaneous fascia was closed by the continuous suture method using absorbable thread (sized 4.0 with a small needle) and ordinary skin suture with nylon thread (sized 4.0) with no drain indwelling. Ambulation was permitted when the patient had no complaint of moderate to severe headache after 2 or 3 days of bed rest, with Foley catheter indwelling after detection of IDT with treatment. Intravenous antibiotics were used for 2 days and oral antibiotics for an additional 3 days. All cases of IDT were evaluated by magnetic resonance imaging (MRI) postoperatively at 


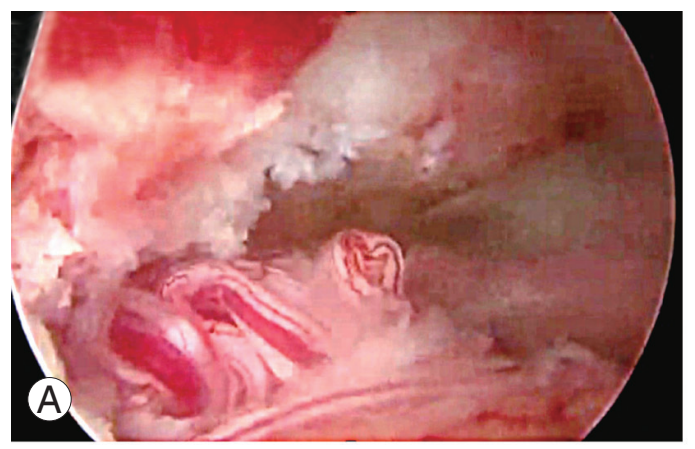

(B)
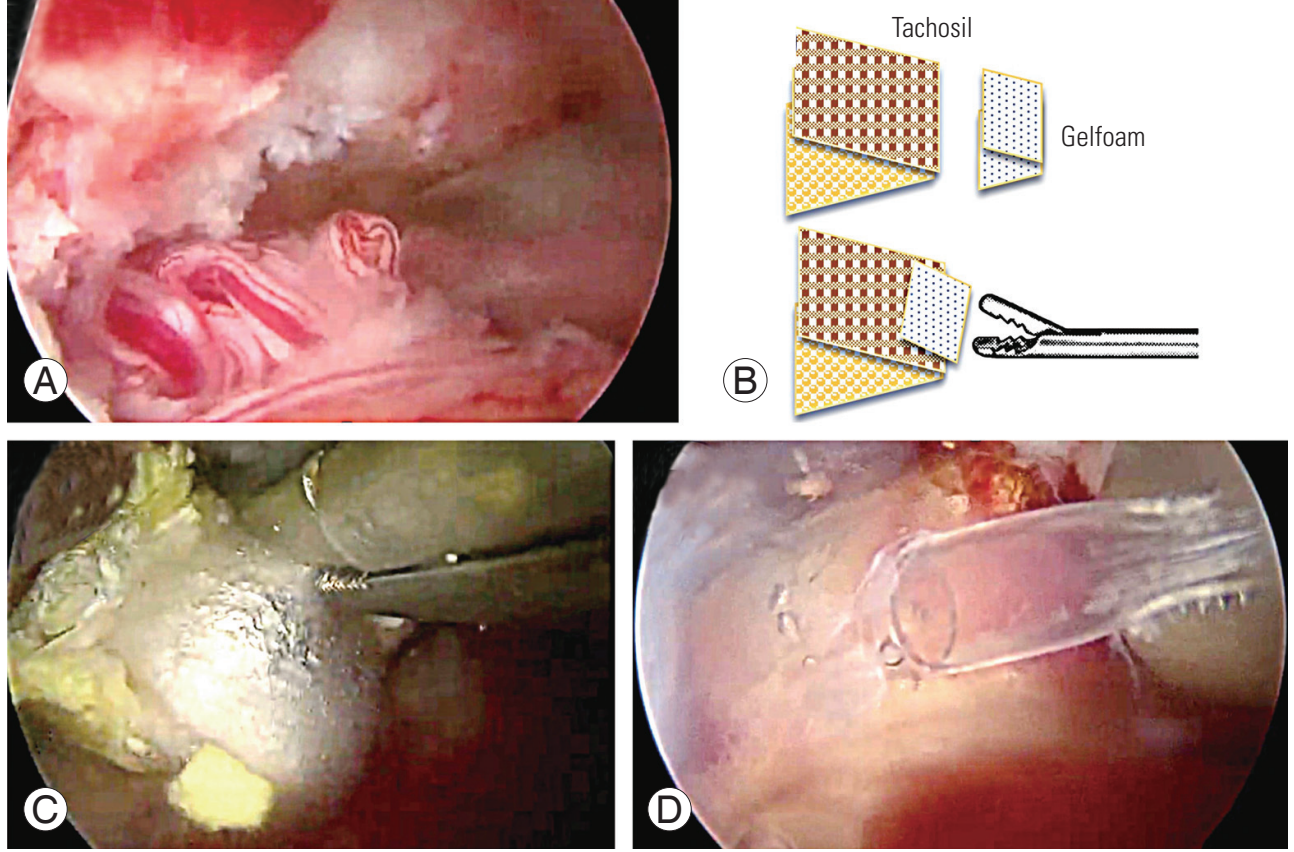

Fig. 1. Patch compression method. (A) Larger slit tear with entrapped rootlets on the contralateral side. (B) TachoSil is divided two pieces and folded about $1 \times 1 \mathrm{~cm}$. A $1 \times 1 \mathrm{~cm}$-sized smooth surfaced Gelfoam is also folded and added over the sticky TachoSil. (C) This helps to grasp and deliver TachoSil into the target area through small portal tract. (D) In this case of a large tear, fibrin glue is also infused to envelop the patched site to decrease dural pulsation and re-herniation of rootlets.

2 or 3 days, 1 week, and 1 month to confirm whether CSF leakage progressed in failed cases or fibrous tissue was covered well and the tear site sealed in successful cases.

\section{Results}

Among 25 total cases of IDT, surgeon 1 had four IDT cases $(0.5 \%)$ out of 768 total patients, surgeon 2 had seven cases (2.8\%) among 253, surgeon 3 had five cases (3.8\%) among of 130 , and surgeon 4 , had nine cases $(2.3 \%)$ among 400. Fourteen cases (56\%) occurred during the early learning-curve period within 6 months after starting BESS. In the early learning period, all surgeons were supposed to experience IDTs at a similar incidence (odd ratio, 0743).

Among the 25 dural tear cases, 14 patients were men and 11 were women, with an average age of $50.5 \pm 13.7$ years. Regarding initial diagnosis, 16 cases were spinal stenosis, six were disc herniation, two were recurred disc herniation, and one was a revision surgery for spinal stenosis (Table 1).

Twenty cases (80\%) of IDT had a tear of $<10 \mathrm{~mm}$ (18 small slit tears and two small flap tears). Of these, 19 cases were improved with conservative treatment using a patch without conversion to open surgery for dural repair. One case was neglected during surgery, and IDT was detected 3 days later via MRI due to clear discharge (CSF leakage) from the wound. This case was treated with delayed open repair at postoperative week 1 after failing blood patch trial twice. Five cases with IDT of $\geq 10 \mathrm{~mm}$ (four large slit tears and one large flap tear) were treated by different methods. Three of four cases of large slit tear underwent immediate conversion to open surgery, and the remaining large slit tear was repaired by the patch compression method. The one large flap tear was managed with patch compression and fibrin glue covering, but it failed so that delayed conversion to open repair was needed due to pseudomeningocele at postoperative week 5 . One case of a large dural tear was sutured by endoscopic clipping without converting to open repair.

IDT mostly occurred during special conditions for specific procedures. Fourteen cases (56\%) of IDT occurred while using a Kerrison rongeur for laminectomy of the ipsilateral or contralateral lower lamina, or flavectomy of a deep layer of ligamentum flavum (LF) at the midline. Five cases occurred while using a curette and two cases while using a burr. Unpracticed handling of muscle serial dilators, slipped tapping of an osteotome, and inattentive 

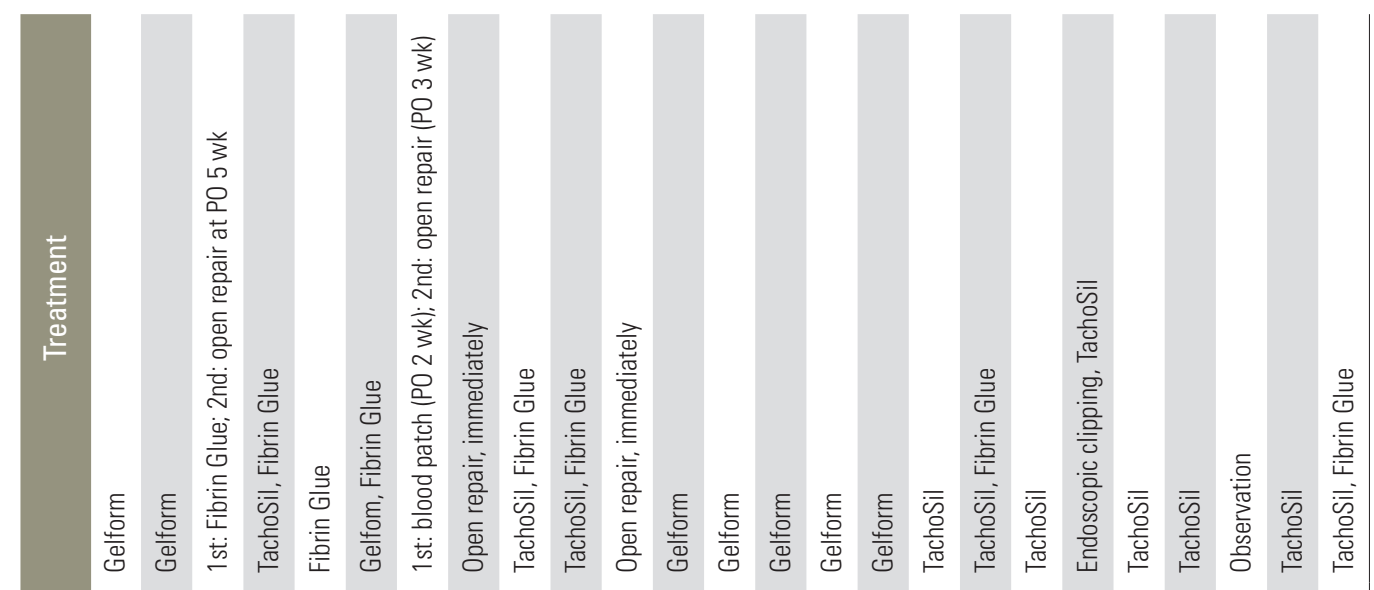

票

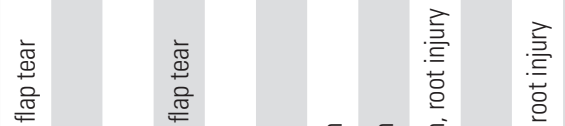

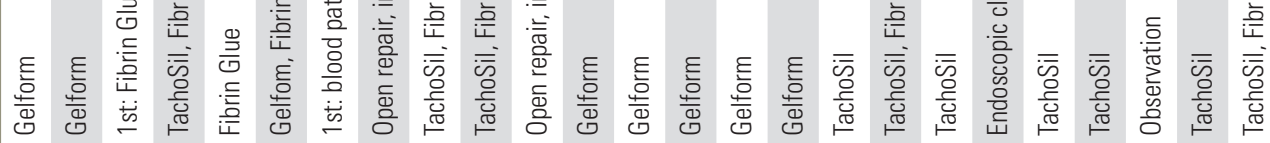

离

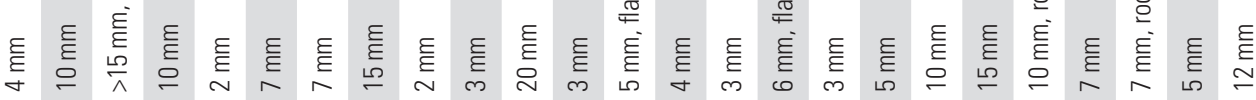

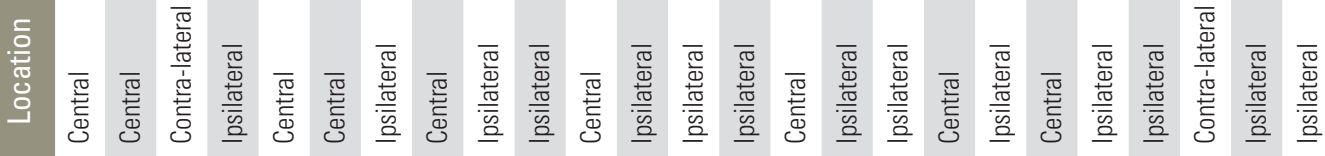

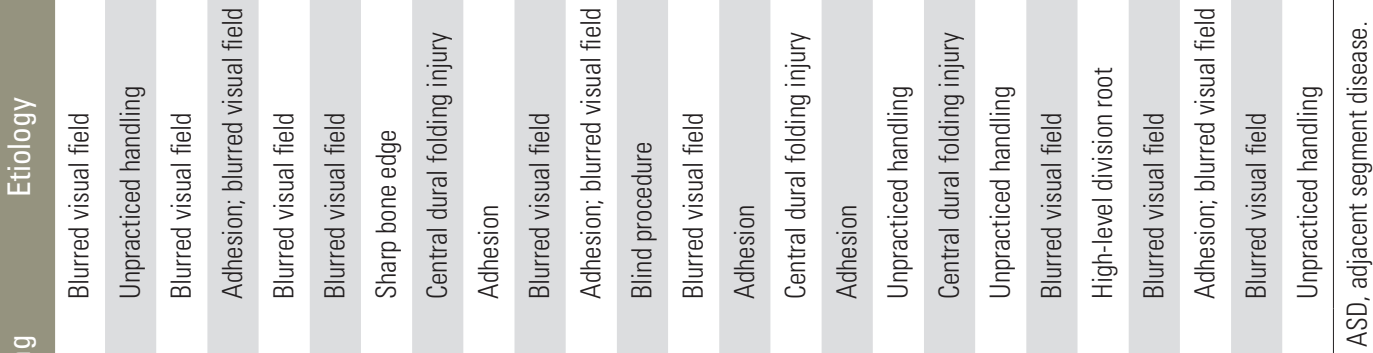

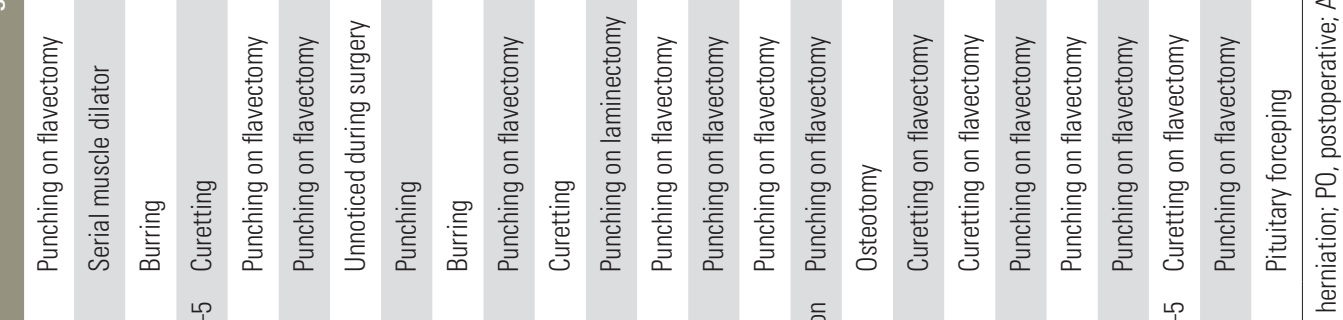

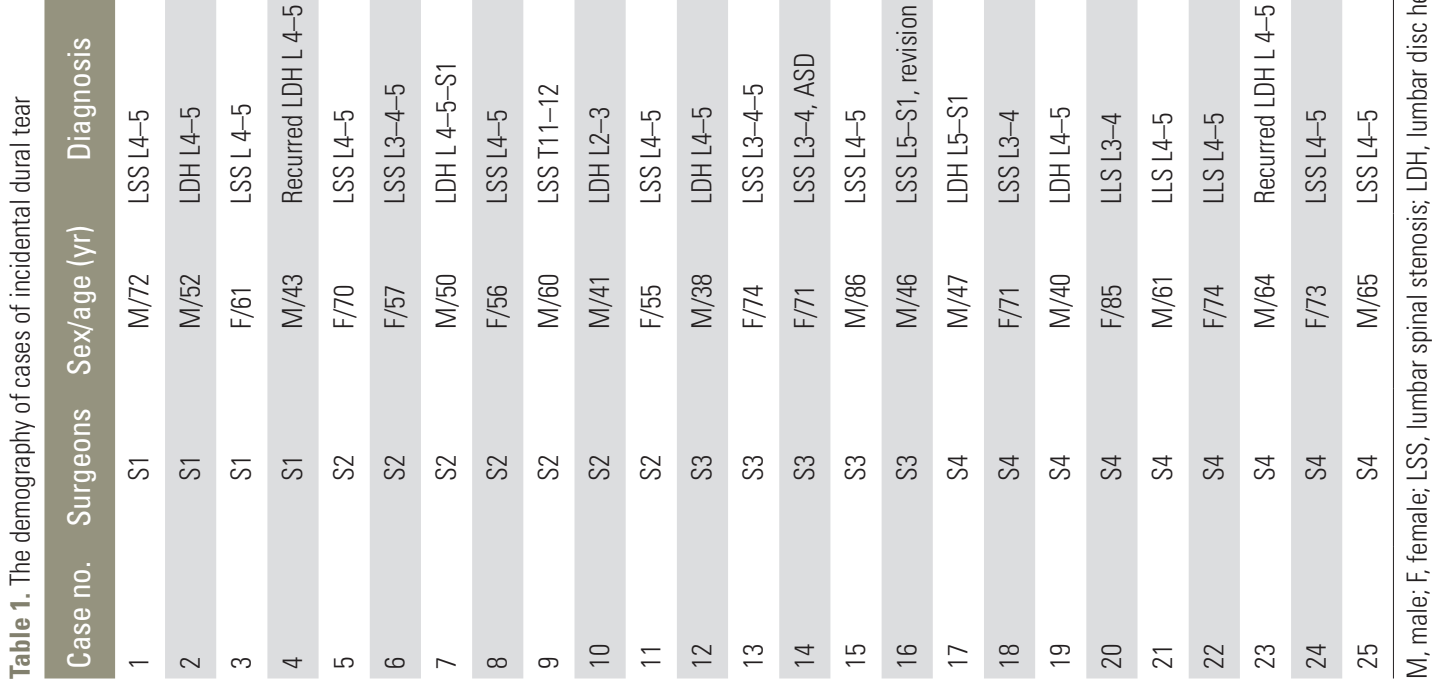


clamping of pituitary forceps were other causes of IDT. Most cases of IDT happened while performing surgery under a blurred visual field obscured by small epidural bleeding or laminectomized cancellous bone. Adhesiolysis of scar tissue using a curette on the dura from the lamina for revision surgery was also one of the causes of IDT. IDTs were commonly located at the central area in nine cases, ipsilateral area in 14 cases, and contralateral area in two cases. In one case, the axillar area at the junction of the dura and root was injured both ipsilaterally and contralaterally. No cases maintained neurologic sequelae or progressed to surgical site infection or infective spondylitis.

\section{Discussion}

BESS is one endoscopic spine surgery using a floating technique a certain distance from the target structures rather than docking into the Kambin's triangle. This permits a foraminal approach and a posterior interlaminar approach, so that the applications of endoscopic spine surgery become wider to include most degenerative spine disorders covered by microscopic spine surgery. BESS was reported to provide similar patient satisfaction, pain improvement, and earlier recovery compared with open microscopic spinal surgery due to the development of improved optics and surgical techniques [1]. Technically, the approach of BESS minimalizes damage to the soft tissue and muscle $[2,3,5,16,17]$.

IDT may lead to incomplete decompression due to a blurred visual field, resulting in poor outcome. In addition, for cases that undergo open conversion, a large incision, paraspinal muscle damage, and delayed recovery is inevitable, leading to a negative short-term postoperative clinical outcome. While some studies report that IDT does not exacerbate clinical results [11,18-20], others have shown poorer results from the patient group that had IDT compared with the group without IDT [21]. Takahashi et al. [22] concluded that IDT risk factors include being female, old age, degenerative spondylolisthesis, juxtafacet cyst, and that IDTs commonly occurr in the cranial, caudal, disc level, and medial side of the facet.

IDT repair via open surgery is one of the most common complications occurring during BESS. The exact incidence rate of IDT after BESS is currently unknown, although a review article reported it as approximately $3 \%$ [2], in contrast to the approximate incidence of micro- scopic lumbar surgery of $8 \%[18,19]$. BESS is performed under water using a floating technique in a certain space through which small bleeds easily blur the surgical field. In the early period when a surgeon learning BESS is not accustomed to the exact depth under an unfamiliar or blurred endoscopic field of view, IDTs easily occur. Thus, the first preventive strategy of IDT is to make a clear surgical field to see the structural margin clearly by keeping the saline output fluent and controlling small bleeds from epidural small vessels by small-headed radiofrequency and laminectomized bone bleeds by sealing with bone wax.

IDTs most commonly occurred in our study during flavectomy in the central area of the spinal canal. The central area of the dura is connected to a few lines of fibrous tissue under the LF, and it is covered and hidden by epidural fat. Infused saline compresses both sides of the dura so that the central of the dura is folded and appears as a central folding. If a central dural folding goes unnoticed while inputting a Kerrsion punch or curette under the epidural fat at the midline for a flavectomy, direct injury of the dura at the midline may occur. To prevent IDT around the central dural folding during flavectomy, instruments should be inserted above the epidural fat layer no further without clear visibility of the structural margins (Fig. 2).

The LF can be divided into two layers: the superficial layer above the lower lamina level and the deep layer at the same and below the level of the lower lamina. To make a sufficient working space and inspect the bony margin, the superficial layer of the LF should be removed before laminectomy. However, removal of the deep layer should be postponed till completion of the laminectomy to protect the dura. This is especially important when using a high-speed burr so that its head does not directly injure the dural membrane. The peripheral fibrous band and vessel bundles of the dura may be dragged and wound around the neck of a rolling burr, causing a larger flap tear. Therefore, we suggest preserving the deep layer of the LF during laminotomy using a burr. If the deep layer of the LF were fully removed before finishing the laminotomy, a piece of Cottonoid could be inserted under the proximal lamina and made long enough to cover the dura briefly while using the burr, especially at the contralateral proximal corner. Any protective Cottonoid covering should be located at least $1 \mathrm{~cm}$ proximally beneath the proximal lamina to prevent it from being swept away by saline flow (Fig. 3). 

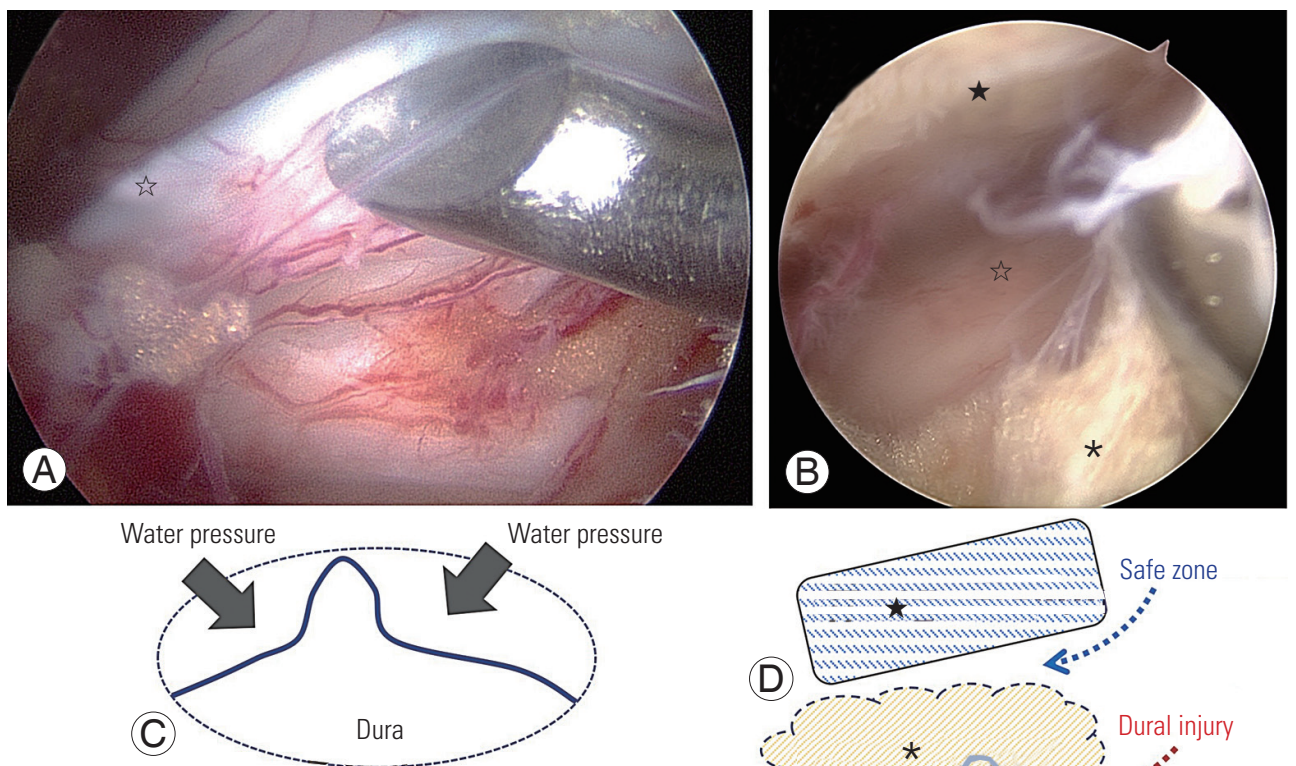

Fig. 2. Central dural folding. (A, B) Intraoperative endoscopic image demonstrating central dural folding. There are three layers: ligamentum flavum $(\star)$, epidural fat layer $(*)$, and central dural folding ( 2$)$. A curette is working on the epidural fat. (C) Effect of water pressure forming the central dural folding. (D) A safe zone for crossing the midline over central dural folding. Insertion of sharp instruments should be avoided under the epidural fat without caution of central dural folding.
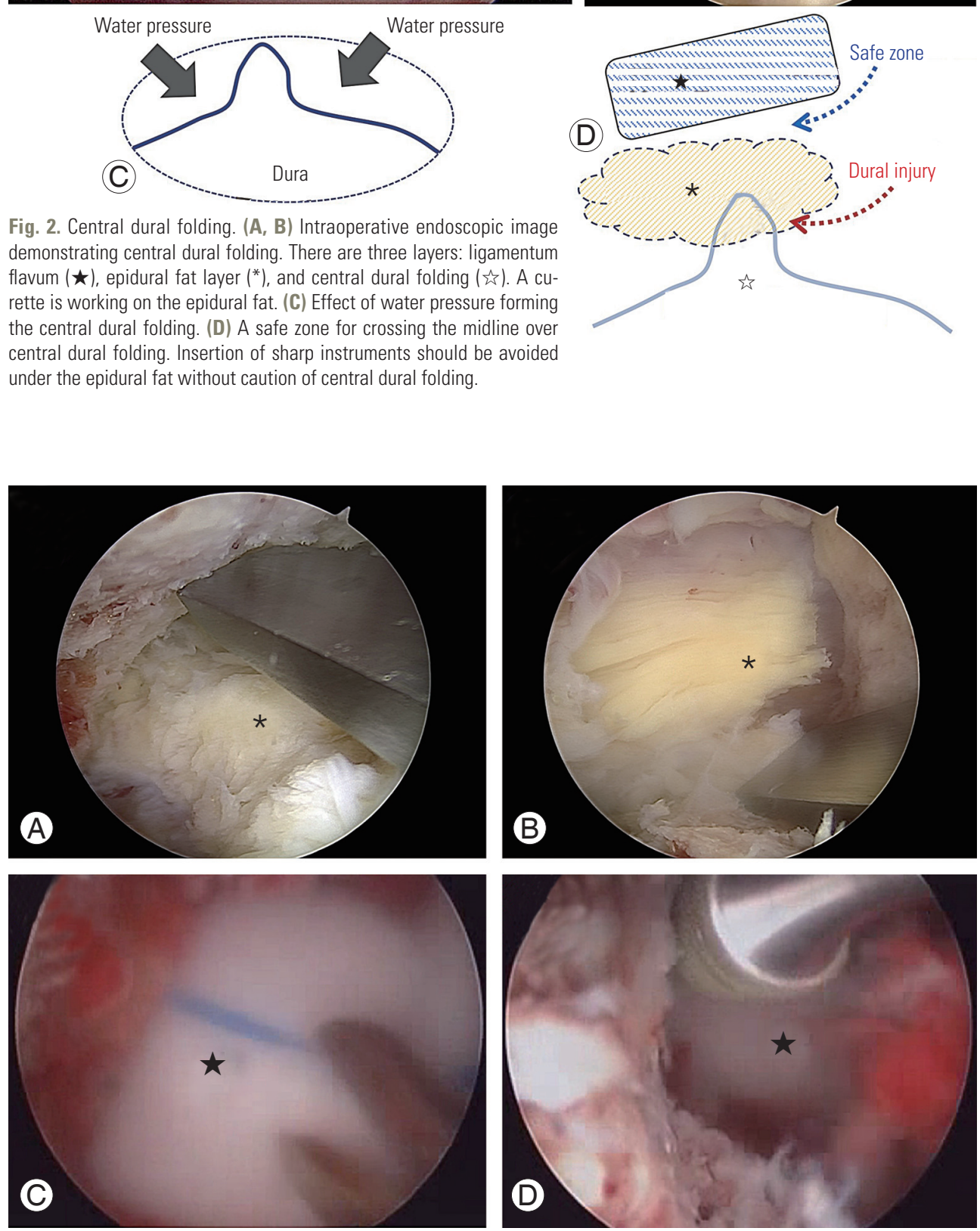

Fig. 3. Laminectomy while protecting the dura. (A, B) Preserving the deep layer of LF until finishing the laminectomy. Contralateral side laminectomy $(A)$ and distal laminectomy $(B)$ were performed with protection of dura by deep layer of $L F\left({ }^{*}\right)$. (C) When the deep layer of LF was detached fully, a Cottonoid covering the dura was inserted under the proximal lamina ( $\star$ ). (D) Burring could be permitted after protection of the dura with Cottonoid carpet $(\star)$. LF, ligamentum flavum. 
One case of unnoticed IDT occurred on a right-side approach. On right side approach without switching hands (left-handed scoping and right-handed instrumentation), right-sided handling of the instrument could be bothered by proximal lamina and spinous process base and make insufficient working space with less amount of laminectomy of the lower lamina or sharp edge. On the surgical video, there was no direct injury of the dural membrane, but dural pulsation after flavectomy could repeatedly macerate the dural membrane on the edge of the lower lamina and delayer a dural tear. Even after two blood patch attempts, CSF still leaked through the portals, necessitating conversion to open surgery identify the cause and tear site. To make enough working space and sufficient decompression on the right-sided approach, portals should be located somewhat lower than the standard position on the left-sided approach, and the medial lamina surface should not be misunderstood as the dorsal lamina, which could bring about insufficient proximal laminectomy and a narrower working space. Forceful handling of instruments and curetting to overcome a narrow working space could leave a sharp bone edge.

IDT could be thought to happen 2 or 3 times more frequently in revision open surgery because of dural adhesion to surrounding tissue [23]. However, there were not enough cases of revision surgery in this study to reveal such a phenomenon. In the early learning-curve period, surgeons prefer to choose a more familiar open technique in complex cases such as severe spinal stenosis or revision surgery so that there could be fewer cases of revision surgery. Therefore, we could not conclude whether fewer IDTs occur with BESS revision surgery due to the higher magnification and illumination benefits of endoscopy [6].

Determining whether an IDT is small or large has not been defined with clinical meaning. We reasoned that a size small enough to prevent rootlet from extruding and being entrapped could be considered "small." Therefore, adding mechanical pressure such as a patch to prevent dural pulsation from making the tear site wider while also encouraging rootlet's out movement can help close the tear space and promote successful healing. Because the dura has a very fast healing rate (about 7 days), perhaps 24-36 hours of tear site stabilization is sufficient for healing $[24,25]$. We further reasoned that a "large" size would be $>1 \mathrm{~cm}$ or flap tear. Dural pulsation would keep the edges of a tear open wide enough for rootlets to extrude easily and be trapped with intolerable pain. Thus, besides closing the opening, preventing dural pulsation must be achieved so that an intentional hematoma compressing the tear site is fostered and not allowed to escape through a drain. Negative pressure by a drain is not helpful for dural healing because it could promote continuous CSF leakage, keeping the tear site open continuously. The clinical results of this study support the definitions of a small-sized tear being $<1 \mathrm{~cm}$ because $95 \%$ of these small tears were treated well with patch compression and 2-day bed rest with no drain. The one failed case is most likely due to omitting additional managements such as a patch, fostered hematoma, and no drain in addition to a sharp edge from an insufficient laminectomy on the right side. Although a blood patch could be one plausible option to prevent CSF leakage in individual cases, it is risky due to the difficulty of accurate positioning to the target area [2628].

In the case of a pseudomeningocele complication, several weeks after surgery a huge flap tear due to burring was noticed on the contralateral side of the laminectomy without protection of the LF on the dura, and revision surgery was mandatory to a debridement pseudomembrane in the subcutaneous and muscular area to find and repair the tear site. But on wider exposure in the revision, the flap-like large tear site was healed spontaneously to a certain amount so that a crescent-like opening of approximately $5 \mathrm{~mm}$ was left just under the proximal lamina corner, so that only a patch was required and resulted in successful healing (Fig. 4).

TachoSil is a fibrin-based hemostat sticky enough to seal the wet surface of the dura. Another piece of Gelfoam is for protecting the TachoSil piece from floating away by dural pulsation or saline flow. Too many pieces of Gelfoam to compress the area are unnecessary and could cause too much compression of the dura, such as symptomatic iatrogenic hematoma on postoperative MRI. Additional fibrin glue was used in six cases of IDT after TachoSil or Gelfoam compression, and in four cases of a large tear with open repair. However, this is supposed not to be needed anymore in later cases of small tears.

Considering these various situations, a new treatment theory and protocol is greatly needed for IDT repair during endoscopic spine surgery. Small-sized IDT of $<1 \mathrm{~cm}$ could be well treated with the patch compression method. If a larger or flap tear of $<10 \mathrm{~mm}$ could be closed with a few stiches using a vascular clip, such cases may be healed by the patch compression method [29]. To do this suc- 
cessfully, three portals are needed for viewing, grasping, and clipping. Therefore, the portal with the very best angle for clipping is reserved for a vascular clip device by the surgeon's dominant hand. Another portal is used for a ring forceps by an assistant hand (Fig. 5).

Even in the case with pseudomeningocele after endo-
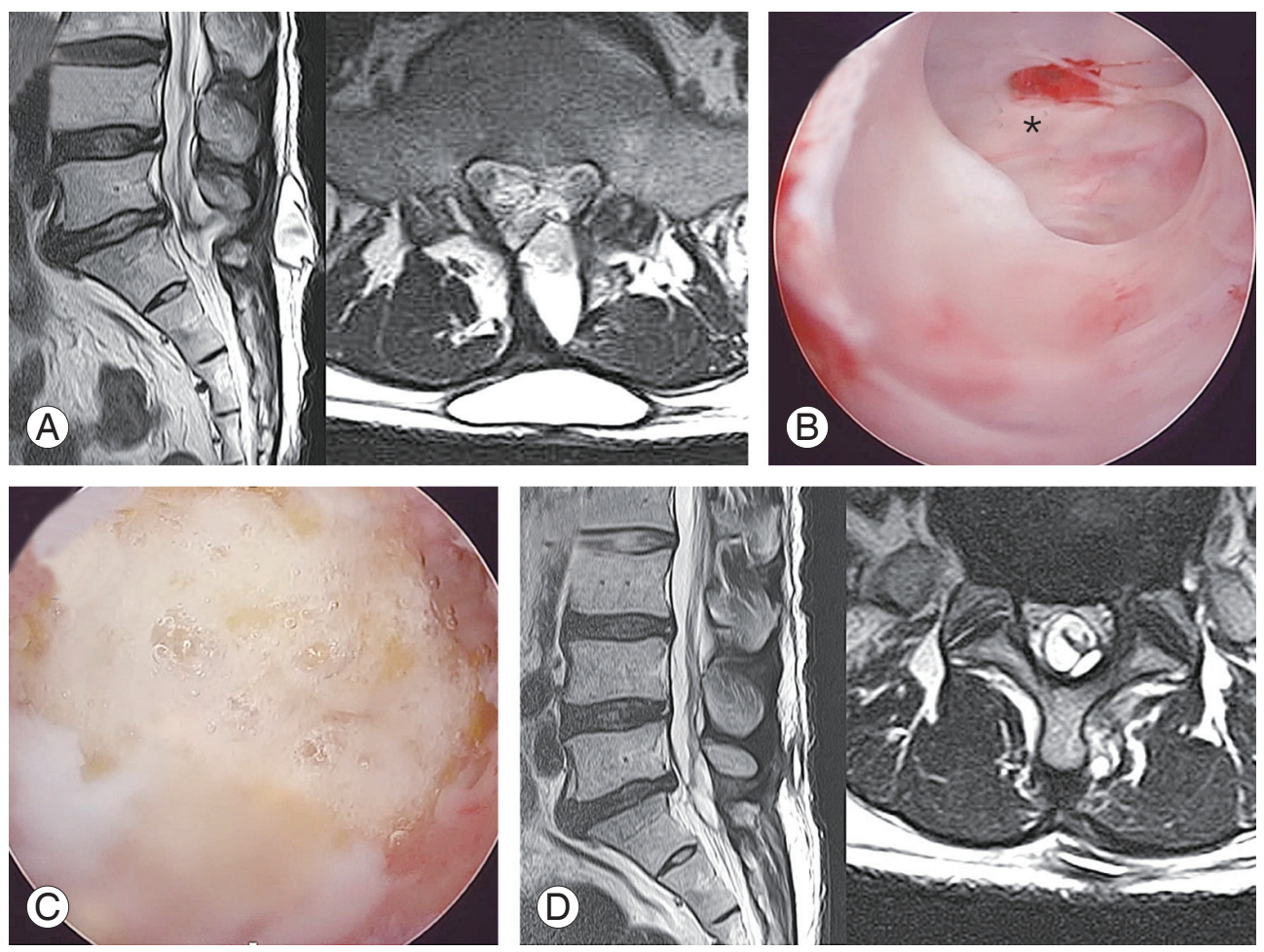

Fig. 4. Pseudomeningocele. (A) A huge pseudomeningocele formed at the subcutaneous layer connecting to the deeper inside of the spinal canal at postoperative week $8 \mathrm{MRI}$. (B) Revision using biportal endoscopic spinal surgery into the pocket for debridement and to eliminate the pseudomembrane and trace the tract into the remnant orifice $\left({ }^{*}\right)$ of the dura. It is already healed and left as a crescent shape. (C) TachoSil is patched at the orifice. (D) Postrevision 4-week MRI shows a successfully resolved state. MRI, magnetic resonance imaging.
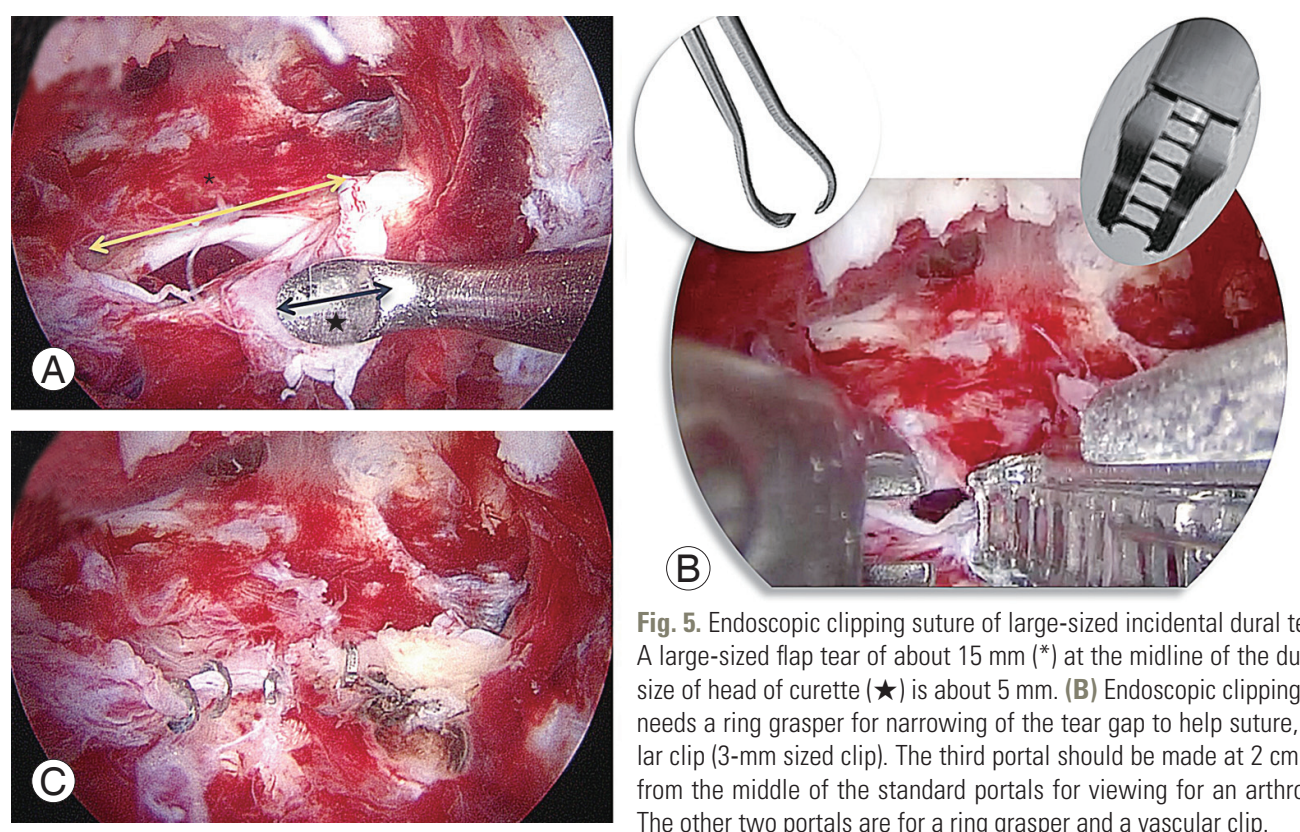

Fig. 5. Endoscopic clipping suture of large-sized incidental dural tear. (A) A large-sized flap tear of about $15 \mathrm{~mm}\left({ }^{*}\right)$ at the midline of the dura. The size of head of curette $(\star)$ is about $5 \mathrm{~mm}$. (B) Endoscopic clipping suture needs a ring grasper for narrowing of the tear gap to help suture, vascular clip (3-mm sized clip). The third portal should be made at $2 \mathrm{~cm}$ lateral from the middle of the standard portals for viewing for an arthroscope. The other two portals are for a ring grasper and a vascular clip. 


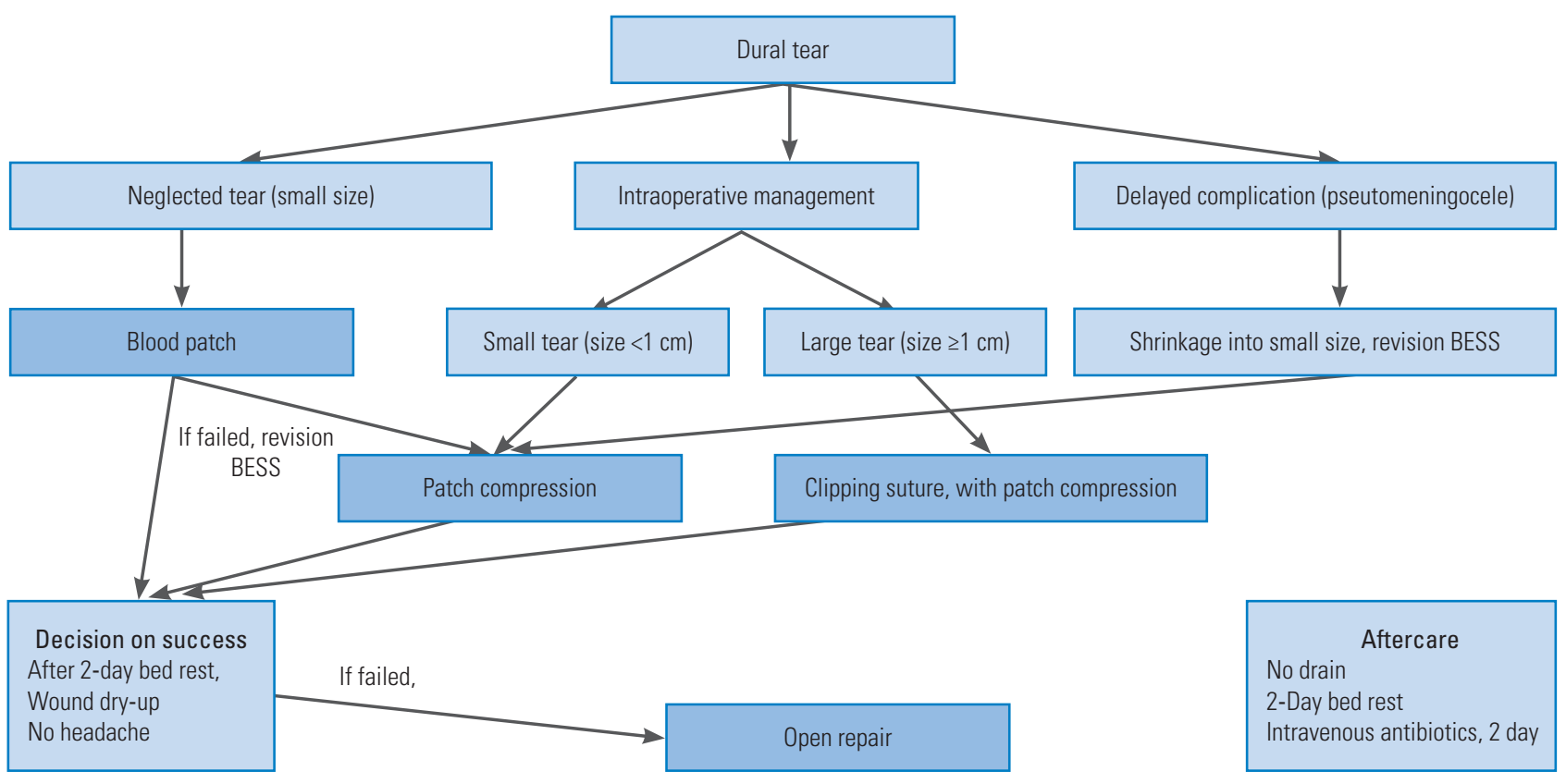

Fig. 6. Diagram of treatment protocol for incidental dural tear during BESS. BESS, biportal endoscopic spinal surgery.

scopic or open spine surgery, revision using BESS could be performed directly in the middle of a pseudocyst at the subcutaneous layer, and following the track of CSF draining could lead us to the tear site, which was already healed to a smaller size adaptable to the patch compression method. Our recommended protocols to manage IDT during BESS are summarized in Fig. 6.

As a standard treatment for IDT during MIS, conversion to open surgery for direct repair has been considered as a mandatory treatment. But this requires sufficient room with much larger muscle dissection and wider laminectomy to handle instruments for delicate suture. This approach could jeopardize the facet stability and cause chronic lower back pain above the dural tear itself. Endoscopic spine surgery becomes more and more popular for wider indications on degenerative spine disorders such lumbar disc herniation, spinal stenosis with degenerative spondylolisthesis, foraminal stenosis, and even revision surgery. Therefore, endoscopic spine surgeons should mandatorily have a reasonable treatment concept and standard protocol to manage IDT during endoscopic procedures.

\section{Conclusions}

IDT during BESS occurred at a frequency of about 1.6\% $(0.5 \%-3.8 \%)$. This could happen during mechanical decompressing procedures especially in the early learning- curve period. A new surgical skill can handicap newly recruited surgeons unfamiliar with endoscopic geometry procedural steps. Therefore, surgical precautions should be kept in mind such as making the surgical view clear with fluent saline outflow and meticulous control of small bleeding and protecting the dura with a deep layer of the LF until finishing laminectomy when punching and curetting are needed. Treatment protocols may include patch compression methods for small-sized dural tears without converting to open surgery or keeping the benefits of minimally invasive spine surgery, while ensuring that larger-sized dural tears of $>1 \mathrm{~cm}$ or flap tears would require an open repair.

\section{Conflict of Interest}

No potential conflict of interest relevant to this article was reported.

\section{References}

1. Min WK, Kim JE, Choi DJ, Park EJ, Heo J. Clinical and radiological outcomes between biportal endoscopic decompression and microscopic decompression in lumbar spinal stenosis. J Orthop Sci 2019:S0949-2658(19)30187-3.

2. Kim JE, Choi DJ, Park EJJ, et al. Biportal endoscopic spinal surgery for lumbar spinal stenosis. Asian Spine 
J 2019;13:334-42.

3. Kim JE, Choi DJ. Clinical and radiological outcomes of unilateral biportal endoscopic decompression by $30^{\circ}$ arthroscopy in lumbar spinal stenosis: minimum 2-year follow-up. Clin Orthop Surg 2018;10:328-36.

4. Choi DJ, Kim JE, Jung JT, et al. Biportal endoscopic spine surgery for various foraminal lesions at the lumbosacral lesion. Asian Spine J 2018;12:569-73.

5. Kim JE, Choi DJ. Biportal endoscopic transforaminal lumbar interbody fusion with arthroscopy. Clin Orthop Surg 2018;10:248-52.

6. Choi DJ, Jung JT, Lee SJ, Kim YS, Jang HJ, Yoo B. Biportal endoscopic spinal surgery for recurrent lumbar disc herniations. Clin Orthop Surg 2016;8:325-9.

7. Choi DJ, Choi CM, Jung JT, Lee SJ, Kim YS. Learning curve associated with complications in biportal endoscopic spinal surgery: challenges and strategies. Asian Spine J 2016;10:624-9.

8. Tafazal SI, Sell PJ. Incidental durotomy in lumbar spine surgery: incidence and management. Eur Spine J 2005;14:287-90.

9. Guerin P, El Fegoun AB, Obeid I, et al. Incidental durotomy during spine surgery: incidence, management and complications: a retrospective review. Injury 2012;43:397-401.

10. Bosacco SJ, Gardner MJ, Guille JT. Evaluation and treatment of dural tears in lumbar spine surgery: a review. Clin Orthop Relat Res 2001;(389):238-47.

11. Cammisa FP Jr, Girardi FP, Sangani PK, Parvataneni HK, Cadag S, Sandhu HS. Incidental durotomy in spine surgery. Spine (Phila Pa 1976) 2000;25:2663-7.

12. Epstein NE. The frequency and etiology of intraoperative dural tears in 110 predominantly geriatric patients undergoing multilevel laminectomy with noninstrumented fusions. J Spinal Disord Tech 2007;20:380-6.

13. Puvanesarajah V, Hassanzadeh $\mathrm{H}$. The true cost of a dural tear: medical and economic ramifications of incidental durotomy during lumbar discectomy in elderly Medicare beneficiaries. Spine (Phila Pa 1976) 2017;42:770-6.

14. Muller SJ, Burkhardt BW, Oertel JM. Management of dural tears in endoscopic lumbar spinal surgery: a review of the literature. World Neurosurg 2018;119:494-9.

15. Soma K, Kato S, Oka H, et al. Influence of incidental dural tears and their primary microendoscopic repairs on surgical outcomes in patients undergoing microendoscopic lumbar surgery. Spine J 2019;19:1559-65.

16. Kim JE, Choi DJ. Unilateral biportal endoscopic decompression by $30^{\circ}$ endoscopy in lumbar spinal stenosis: technical note and preliminary report. J Orthop 2018;15:366-71.

17. Kim JE, Choi DJ, Park EJ. Clinical and radiological outcomes of foraminal decompression using unilateral biportal endoscopic spine surgery for lumbar foraminal stenosis. Clin Orthop Surg 2018;10:439-47.

18. Matsumoto $M$, Hasegawa $T$, Ito $M$, et al. Incidence of complications associated with spinal endoscopic surgery: nationwide survey in 2007 by the Committee on Spinal Endoscopic Surgical Skill Qualification of Japanese Orthopaedic Association. J Orthop Sci 2010;15:92-6.

19. Tsutsumimoto T, Yui M, Uehara M, Ohta H, Kosaku $\mathrm{H}$, Misawa $\mathrm{H}$. A prospective study of the incidence and outcomes of incidental dural tears in microendoscopic lumbar decompressive surgery. Bone Joint J 2014;96-B:641-5.

20. Wang JC, Bohlman HH, Riew KD. Dural tears secondary to operations on the lumbar spine: management and results after a two-year-minimum followup of eighty-eight patients. J Bone Joint Surg Am 1998;80:1728-32.

21. Saxler G, Kramer J, Barden B, Kurt A, Pfortner J, Bernsmann K. The long-term clinical sequelae of incidental durotomy in lumbar disc surgery. Spine (Phila Pa 1976) 2005;30:2298-302.

22. Takahashi Y, Sato T, Hyodo H, et al. Incidental durotomy during lumbar spine surgery: risk factors and anatomic locations: clinical article. J Neurosurg Spine 2013;18:165-9.

23. Kalevski SK, Peev NA, Haritonov DG. Incidental dural tears in lumbar decompressive surgery: incidence, causes, treatment, results. Asian J Neurosurg 2010;5:54-9.

24. Wolff S, Kheirredine W, Riouallon G. Surgical dural tears: prevalence and updated management protocol based on 1359 lumbar vertebra interventions. Orthop Traumatol Surg Res 2012;98:879-86.

25. Albayrak S, Ozturk S, Ayden O, Ucler N. Dural tear: a feared complication of lumbar discectomy. Turk Neurosurg 2016;26:918-21.

26. Dorbad M, Han J, Ahmed S, Monroe B, Entrup M. 
Lumbar epidural blood patch via a caudal catheter after surgical dural tear and failed repair: a case report. A A Case Rep 2017;8:229-31.

27. Wong K, Monroe BR. Successful treatment of postdural puncture headache using epidural fibrin glue patch after persistent failure of epidural blood patches. Pain Pract 2017;17:956-60.

28. Defresen AA, Smal N, Belle FC, Renwart HP, Bonhomme VL. Combined minimally invasive tech- niques to cure accidental dural tears occurring during spine surgery: epidural blood patch associated with cerebrospinal fluid drainage and ventral bed rest. Acta Anaesthesiol Belg 2016;67:143-7.

29. Ito K, Aoyama T, Horiuchi T, Hongo K. Utility of nonpenetrating titanium clips for dural closure during spinal surgery to prevent postoperative cerebrospinal fluid leakage. J Neurosurg Spine 2015;23:8129. 